\title{
Sarcoidosis mimicking cholangiocarcinoma
}
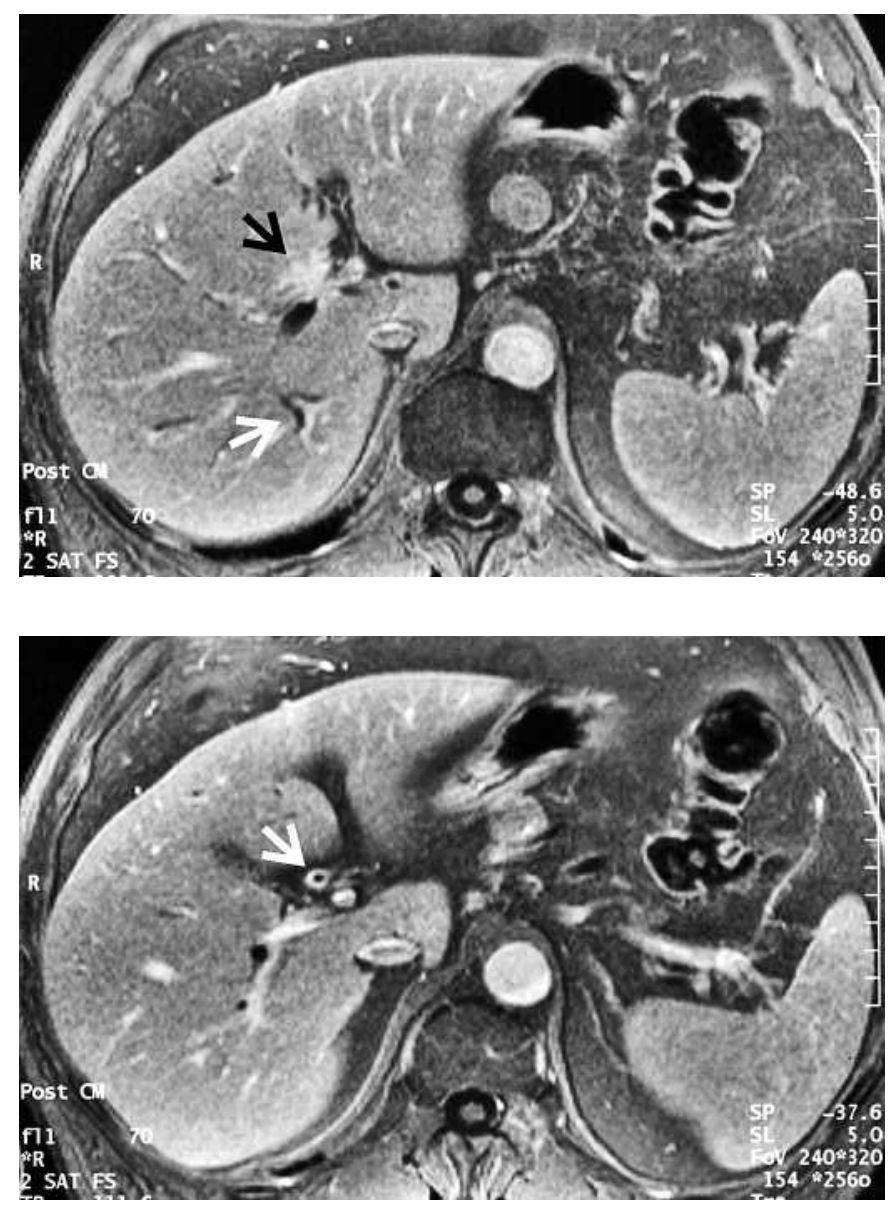

A 68-year-old man was admitted to hospital in May 2002 because of weight loss of $12 \mathrm{~kg}$ within 1 year. Blood tests showed elevated aspartate aminotransferase $224 \mathrm{U} / \mathrm{l}$ (normal range <22), alanine aminotransferase $365 \mathrm{U} / 1$ (normal range <24), alkaline phosphatase 513 U/l (normal range 60 -170), $\gamma$-glutamyl transpeptidase $1.031 \mathrm{U} / 1$ (normal range <28), total serum bilirubin $2.2 \mathrm{mg} / \mathrm{dl}$ (normal range $<1.0$ ), direct serum bilirubin $1.8 \mathrm{mg} / \mathrm{dl}$ (normal range $<1$ ), and serum angiotensin-converting enzyme $21 \mathrm{U} / 1$ (normal range $<20$ ). There were no abnormalities regarding viral hepatitis markers, antibody profiles, or the CA 19-9 value. The patient had been diagnosed with sarcoidosis 5 years previously.
Figure 2 MRI view showing thickening and contrast enhancement of the slightly dilated $(9$ $\mathrm{mm}$ ) common bile duct wall (arrow).

Figure 1 Abdominal magnetic resonance imaging (MRI) showed a hilar-located contrast-enhanced mass in the liver (20 mm diameter; black arrow), causing stricture of the ducti hepatici and intrahepatic cholestasis (white arrow).

Abdominal magnetic resonance imaging (MRI) showed a hilar-located mass in the liver (20 mm diameter; Figure 1) causing stricture of the ducti hepatici and intrahepatic cholestasis. Moreover, thickening along a length of $38 \mathrm{~mm}$ of the slightly dilated $(9 \mathrm{~mm})$ common bile duct wall was found (Figure 2). Endoscopic retrograde cholangiopancreatography (ERCP) showed a hilar infiltration (Figure 3 ) similar to cholangiocarcinoma. Biliary biopsy specimens did not exhibit malignancy. Liver biopsy revealed noncaseating epitheloid cell granulomas (Figure 4), predominantly in the portal area, compatible with sarcoidosis. After dilation of the left hepatic duct stricture the patient was treated with oral prednisolone.

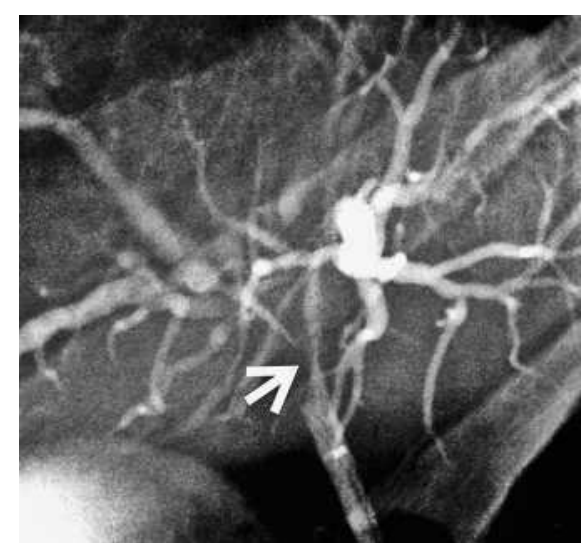

Figure 3 Cholangiogram showing strictures (arrow) of the ductus hepaticus communis (10 $\mathrm{mm}$ length), and both the right and left ducti hepatici $(20 \mathrm{~mm}$ and $10 \mathrm{~mm}$ length, respectively). Note the dilation of the intrahepatic ducts.

At follow-up 6 months later, the pathologic findings from blood tests, MRI and ERCP had resolved (Figure 5), and 47 months later the patient had no clinical signs of malignancy.

Overlap of sarcoidosis and primary biliary cirrhosis or primary sclerosing cholangitis has been described [1,2]. However, clinical signs of bile duct obstruction due to sarcoidosis alone have rarely been reported. Enlarged granulomatous lymph nodes or, as in our patient, masses, are suspected to contribute to biliary obstruction $[3,4]$. Although the combination of both a hilar-located sarcoidoma and an affection of the ductus hepatocholedochus due to sarcoidosis have not been previously reported, it seems likely that the rare feature of biliary obstruction is underreported in this setting. Awareness of sarcoidosis may facilitate its recognition in the differential diagnosis of painless obstructive jaundice. 


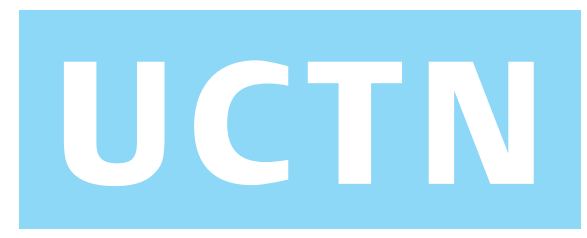

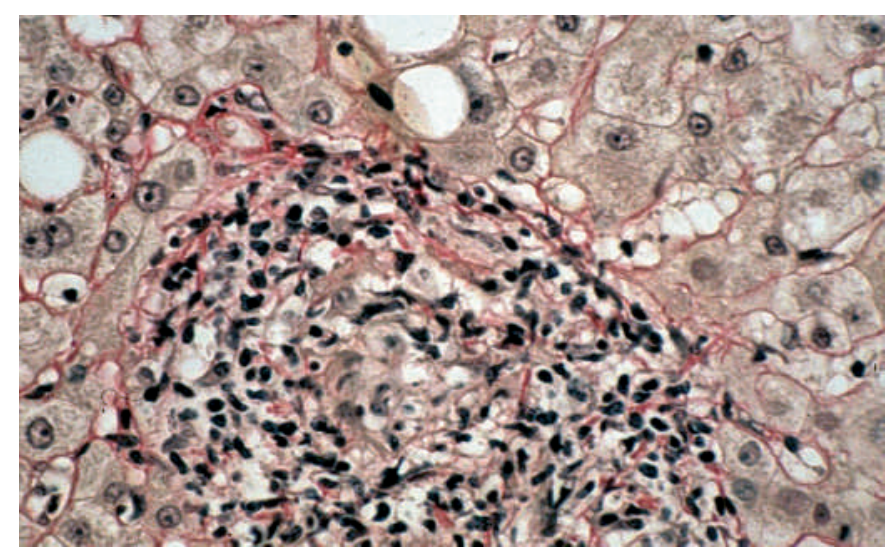

Figure 4 Histopathological examination of the liver biopsy specimen revealed a noncaseating epitheloid cell granuloma.
Endoscopy_UCTN_Code_CCL_1AZ_2AZ

Endoscopy_UCTN_Code_CCL_1AZ_2AC

\section{Nehls, , , H. G. Hass ${ }^{2}$, P. Weber ${ }^{3}$,}

S. Dette ${ }^{1}$, U. M. Lauer ${ }^{1}$, H.-P. Schlemmer ${ }^{4}$,

\section{Oehlert ${ }^{5}$, M. Gregor ${ }^{1}$, B. Klump ${ }^{1}$}

${ }^{1}$ Department of Gastroenterology, Hepatology and Infectious Diseases, Internal Medicine I, University Hospital, Tuebingen, Germany

2 Department of Oncology, Hematology, Immunology, Pulmonology, and Rheumatology, Internal Medicine II, University Hospital, Tuebingen, Germany

${ }^{3}$ Hospital Siloah, Department of Internal Medicine, Pforzheim, Germany

${ }^{4}$ Department of Diagnostic Radiology, University Hospital, Tuebingen, Germany

${ }^{5}$ Institute of Pathology, Freiburg, Germany.

\section{References}

${ }^{1}$ Maddrey WC. Sarcoidosis and primary biliary cirrhosis. N Engl J Med 1983; 308: 588 590

2 Karlish AJ, Thompson RPH, Williams R. A case of sarcoidosis in primary biliary cirrhosis. Lancet 1969; II : 599-

${ }^{3}$ Rezeig M, Fashir BM. Biliary tract obstruction due to sarcoidosis: a case report. Am J Gastroenterol 1997; 92: $527-528$

${ }^{4}$ Baughman R. Sarcoidosis. Usual and unusual manifestations. Chest 1988; 94: 165-170

\section{Corresponding Author}

\section{O. Nehls, M.D.}

Internal Medicine I/II

University Hospital Tuebingen

Otfried-Mueller-Str. 10

Tuebingen 72076

Fax: $\quad+49-7071294457$

E-mail: oliver.nehls@med.unituebingen.de

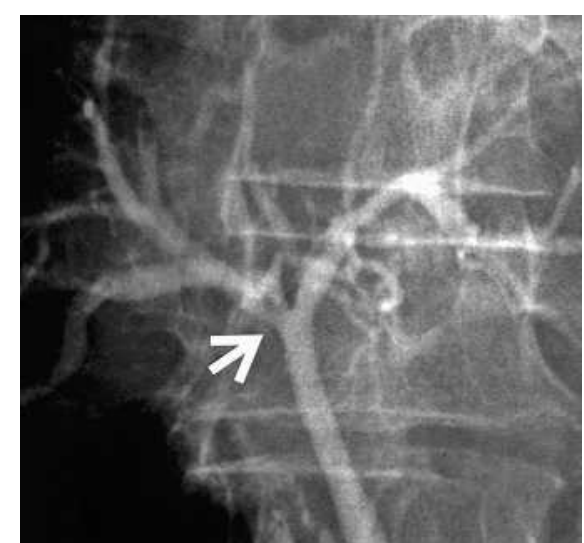

Figure 5 Cholangiography performed 6 months after initiation of treatment with corticosteroids revealed that the pathologic findings (arrow) had been resolved. 